\title{
Impact of mitochondrial DNA mutations in multiple myeloma
}

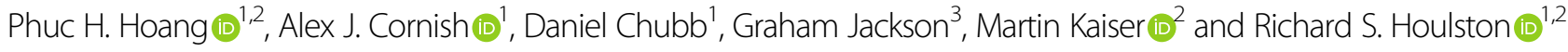

\section{Dear Editor,}

Most cancers have altered metabolism with increased uptake of glucose (i.e. the "Warburg effect") attributed to defective mitochondria ${ }^{1}$. In addition, mitochondria are associated with multiple key processes linked to tumourigenesis including apoptosis, cell cycle, cell growth, and signalling ${ }^{2}$. Multiple myeloma (MM) is essentially an incurable haematological malignancy, with most patients developing resistance to treatment and eventually dying from relapse. Recent studies have proposed mitochondria dysfunction is important in defining chemotherapy resistance and disease progression in $\mathrm{MM}^{3,4}$. Such an assertion is supported by pre-clinical studies, which have suggested agents targeting mitochondria in relapsed MM can improve patient outcome ${ }^{5,6}$. Thus far, the spectrum of mitochondrial DNA (mtDNA) mutations and their functional implications in MM have not however been well characterised, partly due to limited sample size and whole-exome sequencing depth ${ }^{7}$. Furthermore, the paucity of MM representation in pan-cancer analyses ${ }^{7}$ has not allowed an appraisal of MMspecific mitochondrial mutations. By analysing wholegenome sequencing (WGS) data from the Myeloma XI trial, we have sought to address these shortcomings, characterising the somatic mutation landscape, mutation selection at relapse, nuclear genome integration, and copy number of MM mitochondria.

To investigate mtDNA mutations in MM, we analysed WGS data on 80 matched tumour and normal samples from newly diagnosed patients, of which 25 also had matched relapsed tumours. Owing to high cellular copy number of mtDNA genomes, we obtained far greater

\footnotetext{
Correspondence: Richard S. Houlston (Richard.Houlston@icr.ac.uk)

${ }^{1}$ Division of Genetics and Epidemiology, The Institute of Cancer Research, London, UK

${ }^{2}$ Division of Molecular Pathology, The Institute of Cancer Research, London, UK Full list of author information is available at the end of the article
}

mtDNA genome coverage (normals: median $2149 \times$, range $1015 \times-7777 \times$; primary tumours: median $7836 \times$, range $2376 x-7938 \times$; relapsed tumours: median $7826 x$, range $4678 \times-7929 \times$ ) compared to the nuclear genome (Supplementary Table 1).

We identified $210 \mathrm{mtDNA}$ single nucleotide variants (SNVs) in the 80 primary tumours (median 3 SNVs/ tumour). These showed strong replicative strand bias, predominantly $\mathrm{C}>\mathrm{T}$ on heavy strand and $\mathrm{T}>\mathrm{C}$ on light strand (Supplementary Fig. 1), which has previously been ascribed to replication-coupled process partly due to the lack of transcriptional strand bias $^{7}$. Examining the sequence context of mutations revealed the contribution of defective transcription-coupled DNA repair COSMIC signatures 12 (16\%), 21 (15\%), 23 (11\%), and 26 (48\%) (Fig. 1a). We observed transcriptional strand bias across all genes (Fig. 1b), with the strongest signal for $\mathrm{C}>\mathrm{T}$, where transcribed strand are more frequently repaired ${ }^{8}$. The weaker transcriptional strand bias for $\mathrm{T}>\mathrm{C}$ is likely due to the neutralising effects from COSMIC signatures with opposing transcriptional strand biases (Supplementary Fig. 2). To validate these observations, we repeated the analysis of mtDNA mutational spectra using WGS data from 850 newly diagnosed $\mathrm{MM}^{9,10}$ generated by The Relating Clinical Outcomes in Multiple Myeloma to Personal Assessment of Genetic Profile Study (CoMMpass; tumour and normal sample median read depth of $869 \times$ and $661 \times$, respectively). The mutational spectra and strand biases observed in the Myeloma XI samples were also apparent in CoMMpass (Supplementary Fig. 3). Transcriptional strand biases in the CoMMpass samples persist when considering the 22 tRNA genes (14 light strand and 8 heavy strand) separately (Supplementary Fig. 3d). Collectively, these findings are consistent with the contribution of transcription-coupled DNA repair defects in $\mathrm{MM}$ mtDNA. 
a

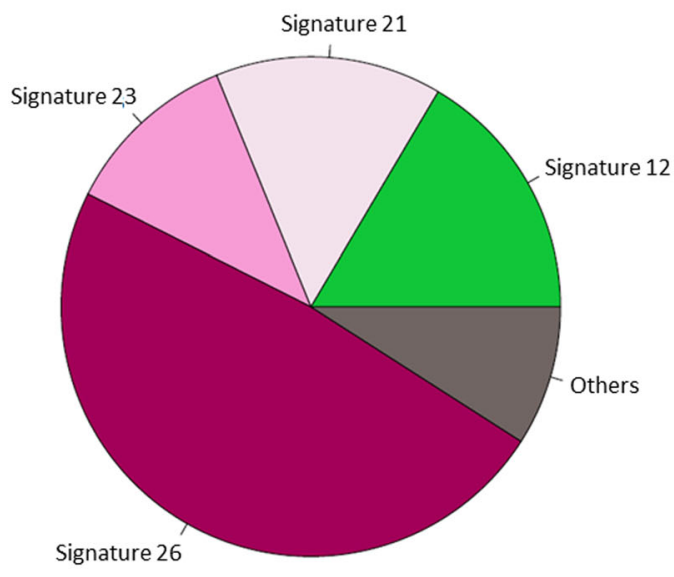

\section{b}

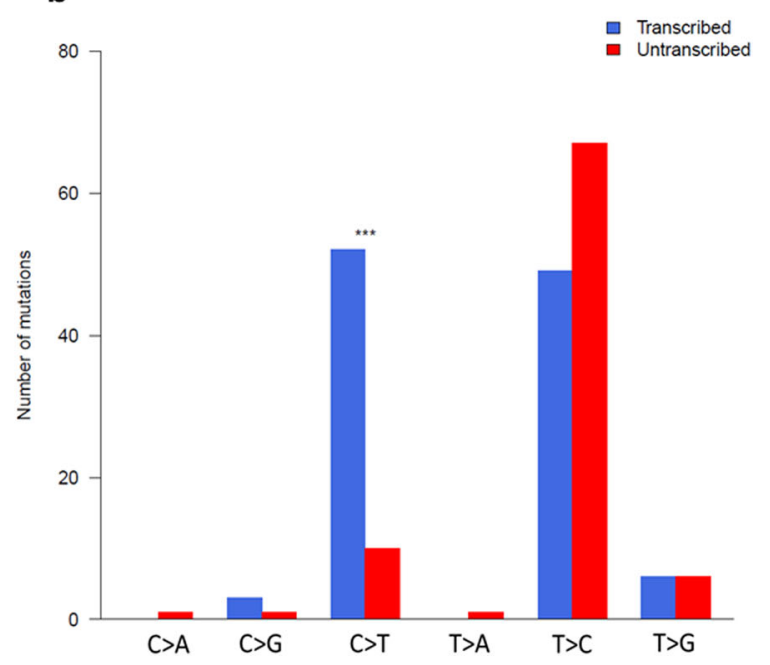

Fig. 1 Mutational signatures in mitochondrial DNA of $\mathbf{8 0}$ primary tumours from Myeloma XI trial. a Contribution of COSMIC mutational signatures extracted by deconstructSigs. $\mathbf{b}$ Transcriptional strand biases across all mitochondrial genes. Significant difference in strand bias was assessed by proportion tests. ${ }^{* *} P<0.01,{ }^{* *} P<0.001$.

Within the 80 Myeloma XI trial samples, 14/210 (6\%) of somatic mutations were identified as being pathogenic (Supplementary Table 2); a number being associated with established diseases ${ }^{11}$ including m.4136A $>\mathrm{G}$ (Leper's optic atrophy), m.9185T $>C$ (Charcot-Marie-Tooth disease, Leigh syndrome, complex $\mathrm{V}$ deficiency), m.15246G >A (development delay, hearing impairment, macrocephalus), and $\mathrm{m} .15287 \mathrm{~T}>\mathrm{C}$ (familial breast cancer). As mitochondrial disease is rare in the general population (around 1 in 5000$)^{12}$, it is likely these mutations have a direct effect on gene function.

We did not observe significant difference in mtDNA somatic mutational burden between MM subtypes, or between primary and relapse tumours (Supplementary Fig. 4). Most germline variants are homoplasmic, whereas somatic variants are more variable in their heteroplasmic level $\left(P<2.2 \times 10^{-16}\right.$, Wilcoxon rank-sum test) (Supplementary Fig. 5). The majority of germline mutations are located outside protein-coding regions or synonymous mutations, with no loss-of-function (i.e. truncating) variants detected (Fig. 2a). In contrast, somatic mutations are more enriched for missense and truncating variants $(P<$ $2.2 \times 10^{-16}$ ) (Fig. 2a), suggesting germline and somatic variants are under different selection constraints. The most frequently disrupted mtDNA coding genes by nonsynonymous somatic mutations include MT-ND5 (29\% of primary tumours), MT-ND4 (24\%), MT-CO1 (20\%), and MT-ND1 (15\%) (Supplementary Table 3).

The $\mathrm{d} N / \mathrm{d} S$ ratio provided no evidence of positive or negative selection for somatic mutations in primary tumours ( $\mathrm{d} N / \mathrm{d} S=1.24,95 \% \mathrm{CI}$ : 0.76-2.03; $P=0.39$ ) (Fig. $2 \mathrm{~b})$, consistent with the observation that missense and truncating mutations do not have significantly different heteroplasmic levels compared to silent mutations (Fig. 2c). However, non-synonymous mutations were positively selected at relapse $(\mathrm{d} N / \mathrm{d} S$ ratio $3.01,95 \%$ CI: $1.09-8.25$; $P=0.033$ ) (Fig. 2b), in concordance with significant increase in homoplasmy of non-synonymous mutations at relapse (Supplementary Fig. 6). Notably, missense mutations in mitochondrial genes composed of the NADH dehydrogenase complex (MT-ND2, MT-ND4, and MT$N D 5)$, feature a higher than expected rate of missense mutations (i.e. positively selected) at relapse $(Q<0.05)$ (Fig. 2d), with non-synonymous mutations in MT-ND5 and $M T-C O 3$ being most frequently acquired at relapse (Supplementary Table 4). These findings imply potential survival advantage rendered through disruption of these genes.

We next sought to examine the effects of mtDNA copy numbers and somatic transfer in MM. We did not find significant difference between mtDNA copy number of tumours and their matched normal, relapsed tumours versus primary tumours, or between high- and low-risk MM subtypes (Supplementary Fig. 7). The results therefore do not support pathogenic and prognostic contribution of mtDNA copy number in MM.

We observed 11/80 primary tumours and 6/25 relapsed tumours positive for somatic transfer of mtDNA to nuclear DNA (Supplementary Table 5). Transfer breakpoints disrupt open reading frames of known oncogenes including CENPP, FOXK1, MGAT5, ST8SIA1, and RAB4A, suggesting a potential role in $M M$ tumourigenesis.

We present here the mtDNA mutational spectrum of $\mathrm{MM}$, the potential underlying mutational processes, and 


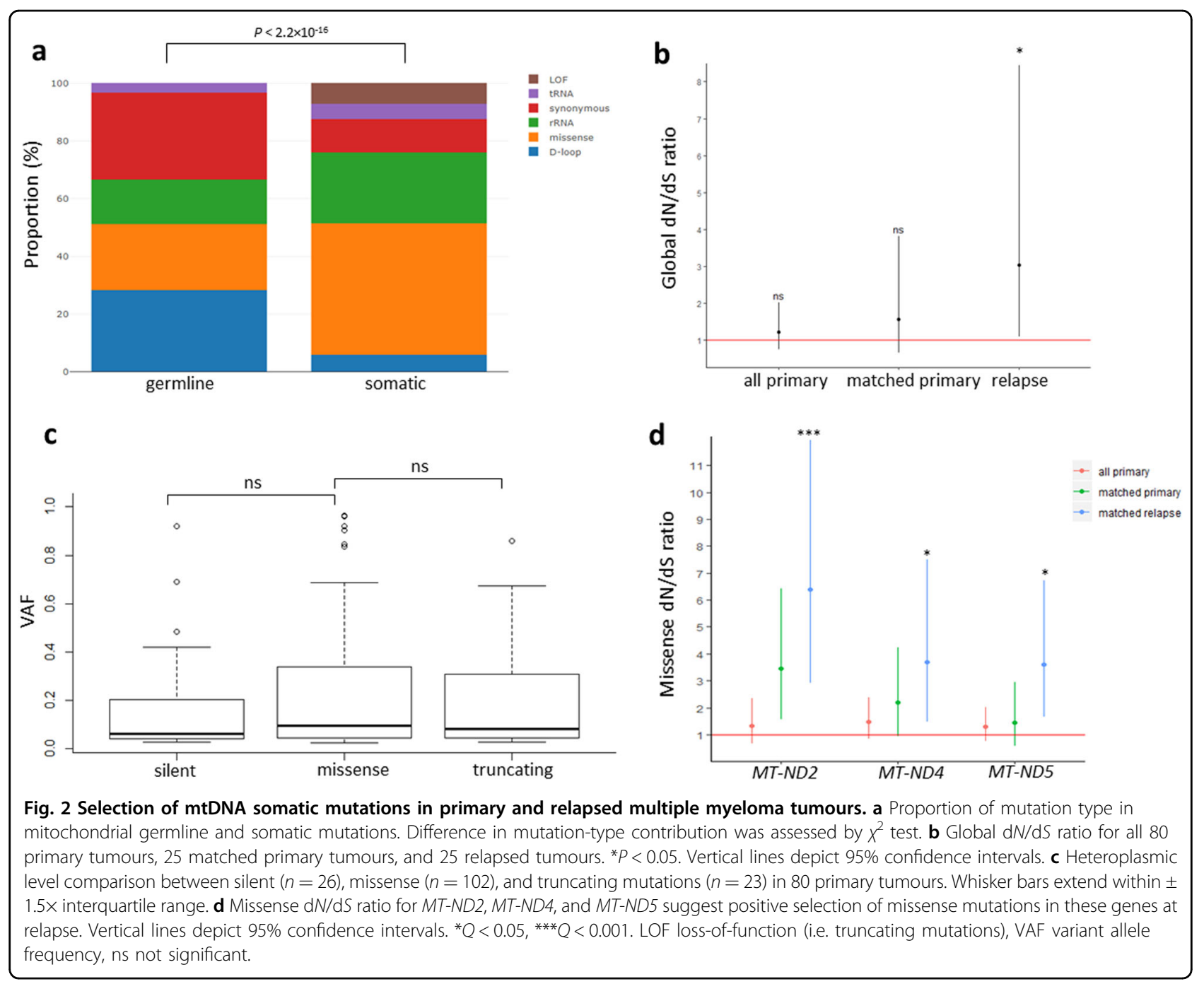

mechanisms in which they could contribute to MM development. We observed transcriptional strand bias of somatic mutations, suggesting transcription-coupled DNA repair defects as one of the main contributing mutational processes in MM mtDNA. This observation is consistent with mitochondria having reduced DNA repair pathways ${ }^{13}$. A larger cohort would be required to unambiguously deconvolve the contribution of each mutational signature at higher nucleotide context resolution. As different defective transcription-coupled DNA repair processes have opposing transcriptional strand biases ${ }^{8}$ and their contribution are varied across tumour types, the transcriptional strand bias might have been neutralised in a previous pan-cancer analysis ${ }^{7}$.

We did not find evidence supporting either negative or positive selection in primary tumours. However, our results do support positive selection at relapse, potentially providing survival and resistance advantage for $\mathrm{MM}$ tumours. Consistent with this, we observed significant $\mathrm{d} N / \mathrm{d} S$ ratio for missense mutations for genes comprising complex I (MT-ND2, MT-ND4, and MT-ND5) and mutations disrupting MT-ND5 and MT-CO3 (cytochrome $c$ oxidase) are frequently acquired at relapse. Functional studies have suggested mutations impacting mitochondrial genes can recapitulate the Warburg effect and provide an alternative mechanism for tumour growth ${ }^{14}$. Although mtDNA copy numbers do not have pathogenic or prognostic implication in MM, mitochondria-nuclear genome integration could potentially contribute to tumourigenesis through disruption of oncogenic genes (e.g. CENPP, FOXK1, MGAT5, ST8SIA1, and RAB4A).

In summary, our study provides evidence to support mitochondrial mutations disrupting electron transport chain, providing potential growth and resistance at relapse MM. Further studies are required to examine the clinical value of mitochondrial mutations as biomarkers, and explore the therapeutic potential of targeting dysregulated metabolism in MM. 


\section{Acknowledgements}

This work was supported by grants from Myeloma UK, Bloodwise, and Cancer Research UK (C1298/A8362). We are grateful to the NCRI Haemato-oncology subgroup and to all investigators for recruiting patients to Myeloma XI. These data were generated as part of the Myeloma UK XI trial and Multiple Myeloma Research Foundation Personalized Medicine Initiatives (https://research. themmrf.org and www.themmrf.org). M.K. is supported by a fellowship from the David Forbes-Nixon Foundation.

\section{Author details}

'Division of Genetics and Epidemiology, The Institute of Cancer Research, London, UK. ${ }^{2}$ Division of Molecular Pathology, The Institute of Cancer Research, London, UK. ${ }^{3}$ Department of Haematology, University of Newcastle, Newcastle Upon Tyne, UK

\section{Author contributions}

P.H.H., A.J.C., and R.S.H conceived and designed the study; P.H.H. performed bioinformatics and statistical analysis; D.C. pre-processed the data; G.J. and M.K. acquired samples; P.H.H. and R.S.H. wrote the manuscript with contributions from A.J.C, D.C., and M.K. All authors reviewed the final manuscript.

\section{Conflict of interest}

The authors declare that they have no conflict of interest.

\section{Publisher's note}

Springer Nature remains neutral with regard to jurisdictional claims in published maps and institutional affiliations.

Supplementary Information accompanies this paper at (https://doi.org/ 10.1038/541408-020-0315-4).

Received: 10 January 2020 Revised: 27 March 2020 Accepted: 20 April 2020 Published online: 01 May 2020

\section{References}

1. Vander Heiden, M. G., Cantley, L. C. \& Thompson, C. B. Understanding the Warburg effect: the metabolic requirements of cell proliferation. Science $\mathbf{3 2 4}$ 1029-1033 (2009).

2. Hengartner, M. O. The biochemistry of apoptosis. Nature 407, 770-776 (2000).

3. Song, I. S. et al. Mitochondrial modulation decreases the bortezomibresistance in multiple myeloma cells. Int. J. Cancer 133, 1357-1367 (2013).

4. Zhan, $X$. et al. Alteration of mitochondrial biogenesis promotes disease progression in multiple myeloma. Oncotarget 8, 111213-111224 (2017).

5. Chanan-Khan, A. A., Borrello, I., Lee, K. P. \& Reece, D. E. Development of targetspecific treatments in multiple myeloma. Br. J. Haematol. 151, 3-15 (2010).

6. Bahlis, N. J. et al. Feasibility and correlates of arsenic trioxide combined with ascorbic acid-mediated depletion of intracellular glutathione for the treatment of relapsed/refractory multiple myeloma. Clin. Cancer Res. 8, 3658-3668 (2002).

7. Ju, Y. S. et al. Origins and functional consequences of somatic mitochondrial DNA mutations in human cancer. Elife 3 (2014).

8. Alexandrov, L. B. et al. The repertoire of mutational signatures in human cancer. Nature 578, 94-101 (2020).

9. Hoang, P. H., Cornish, A. J., Dobbins, S. E., Kaiser, M. \& Houlston, R. S. Mutational processes contributing to the development of multiple myeloma. Blood Cancer J. 9, 60 (2019).

10. Hoang, P. H. et al. Whole-genome sequencing of multiple myeloma reveals oncogenic pathways are targeted somatically through multiple mechanisms. Leukemia 32, 2459-2470 (2018).

11. Shen, L. et al. MSeqDR: a centralized knowledge repository and bioinformatics web resource to facilitate genomic investigations in mitochondrial disease. Hum. Mutat. 37, 540-548 (2016).

12. Gorman, G. S. et al. Prevalence of nuclear and mitochondrial DNA mutations related to adult mitochondrial disease. Ann. Neurol. 77, 753-759 (2015).

13. Alexeyev, M., Shokolenko, I., Wilson, G. \& LeDoux, S. The maintenance of mitochondrial DNA integrity-critical analysis and update. Cold Spring Harb. Perspect. Biol. 5, a012641 (2013).

14. Park, J. S. et al. A heteroplasmic, not homoplasmic, mitochondrial DNA mutation promotes tumorigenesis via alteration in reactive oxygen species generation and apoptosis. Hum. Mol. Genet. 18, 1578-1589 (2009). 